\title{
A Korean case of neurofibromatosis type 1 with an exonic splicing enhancer site mutation
}

\author{
Sangwook Park', Young Bae Sohn², In-Soon Chung ${ }^{2}$, Ji-Hee Hong ${ }^{2}$, Eun-Jung Jung ${ }^{2}$, Seon-Yong Jeong ${ }^{2}$, and Hyun-Seok Jin ${ }^{2}$ * \\ Departments of ${ }^{1}$ Pharmacology and ${ }^{2}$ Medical Genetics, Ajou University School of Medicine, Suwon, Korea
}

\begin{abstract}
Neurofibromatosis type 1 (NF1) is an autosomal dominant disease characterized by neurological, cutaneous, and ophthalmological manifestations. A 33-year-old woman with typical symptoms of NF1 visited Ajou University Hospital. Screening of the whole-messenger RNA region of NF1 at the complementary DNA level by polymerase chain reaction-direct sequencing confirmed the presence of an NF1 mutation at the genomic level. The mutation analysis revealed an in-frame skipping of exon 46 (c.6757_6858del) caused by a point mutation (c. 6792C>A) in exon 46. In this report, we have described the first Korean case of a proband with NF1 that carries an allele with an exon 46 deletion caused by an exonic splicing enhancer site mutation, leading to the skipping of the whole of exon 46 (c.6757_6858del).
\end{abstract}

Key words: Neurofibromatosis 1, Exon skipping, Exonic splicing enhancer, Mutation.

\section{Introduction}

Neurofibromatosis type 1 (NF1; OMIM\# 162200) is an autosomal dominant inherited disorder in humans characterized by café-au-lait spots and fibromatous skin tumors. NF1 is caused by a mutation in the neurofibromin gene (NF1; NM_000267.3) on chromosome 17q11.2, with approximately 60 exons spanning $>350 \mathrm{~kb}$ of genomic DNA (gDNA), transcribing to an messenger RNA (mRNA) of size 11-13 kb [1]. The global incidence of NF1 has been reported to be approximately 1 in 3,000 individuals [2]. Several NF1 symptoms are more common in a proband than in its affected relatives. The maximum incidence of NF1 manifestations tends to increase with age [3]. One of the most common molecular defects of NF1 is predicted to be a protein truncation that alters mRNA splicing. The majority of splicing mutations of NF1 in the conserved splicing site of an intron results in production of aberrant proteins. An exonic splicing enhancer (ESE), which is a DNA sequence motif with 6 bases and an exon, can lead to exon skipping and the production of nonfunctioning proteins. In this study, we have described the first case of a Korean patient possessing an allele with an exon-46 deletion in the NF1 in the mutation of the ESE site, which has been previously reported as c.6792C >A (p. Tyr2264*) [4].

\section{Case}

A 33-year-old woman visited Ajou University Hospital with a history of multiple café-au-lait spots and skin neurofibromas. She had a family history of NF1; her grandfather, mother, aunt, and younger sister had been diagnosed with NF1 (Fig. 1). Her growth and development were normal, and she had no history of surgery. However, physical examination revealed multiple café-au-lait spots, axillary freckling, and multiple skin neurofibromas. The patient did not have plexiform neurofibroma. Ophthalmological examination of the eyes showed bilateral

Received: 2 June 2014, Revised: 9 June 2014, Accepted: 11 Jun 2014, Published: 30 June 2014

*Corresponding author: Hyun-Seok Jin, Ph.D.

Department of Medical Genetics, Ajou University School of Medicine, 164 WorldCup-ro, Yeongtong-gu, Suwon 443-749, Korea.

Tel: +82-31-219-4520, Fax: +82-31-219-4521, E-mail: jinhs@ajou.ac.kr

Conflict of interest: We declare that we do not have any conflicts of interests.

(c) This is an open-access article distributed under the terms of the Creative Commons Attribution Non-Commercial License (http://creativecommons.org/licenses/by-nc/3.0/) which permits unrestricted non-commercial use, distribution, and reproduction in any medium, provided the original work is properly cited.

(c) Copyright 2014 by the Korean Society of Medical Genetics 
Lisch nodules, but no optic nerve glioma.

With a family history of NF1, genetic mutation screening was performed for NF1. Briefly, fresh peripheral blood was collected in ethylenediaminetetraacetic acid (EDTA), and total RNA was extracted from the blood sample by using the OlAamp RNA Blood Kit (Qiagen, Valencia, CA, USA), following the manufacturer's instructions. The isolated total RNA and random hexamers were used to perform reverse transcriptasepolymerase chain reaction (PCR) by using the First-Strand cDNA Synthesis Kit (Fermentas, Hanover, MD, USA). The entire coding

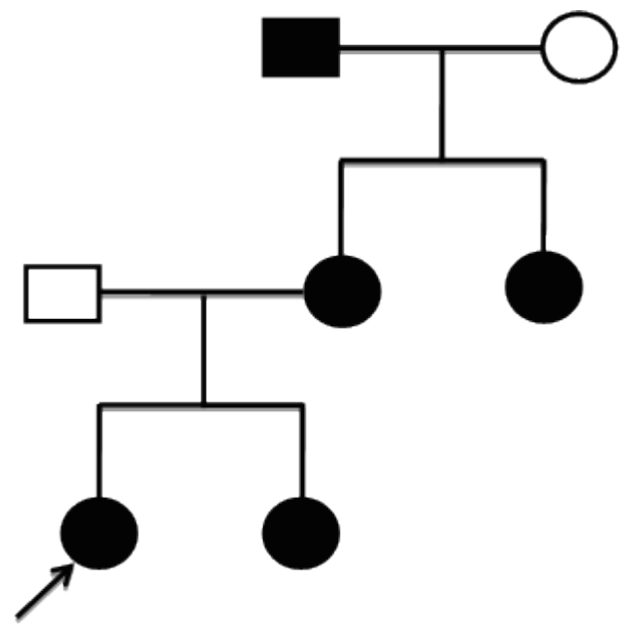

Fig. 1. Pedigree of the neurofibromatosis type 1 (NF1) patient with c.6792C $>$ A mutation. The filled symbols represent the affected patient. The arrow shows the proband. region of NF1 was amplified in 10 overlapping fragments by PCR. Next, the identified mutations were confirmed in the gDNA through direct cycle sequencing by using primers and conditions for genomic sequence amplification (the primer sequences and conditions are available upon request). The PCR product was then sequenced on the ABI 3500xL DNA Analyzer (Applied Biosystems, Foster City, CA, USA).

When a skipped exon was detected at the complementary DNA (cDNA) level, the corresponding exonic and intronic boundaries were sequenced in order to identify the potential mutation. At the cDNA level, we identified an in-frame skipping of exon 46 (c.6757_6858del). In addition, gDNA analysis revealed a point mutation (c.6792C $>$ A) that induced nonsense-mediated residues (p.Tyr2264*) (Fig. 2). A mutation at position c.6792C >A has also been reported previously [5]. We could not examine the mutations in other family members.

\section{Discussion}

To date, 1,699 NF1 mutations have been documented in the Human Gene Mutation Database (http://www.hgmd. cf.ac.uk). The majority of included mutations are truncated NF1 in early decay proteins. Approximately one-third of these aberrant proteins from NF1 are expected to be an RNA-splicing abnormality that often causes exon skipping. Here, we have
A

$\begin{array}{ccc}\text { Exon 46 } & \text { c.6757_6858del } & \text { cDNA level } \\ \text { Normal allele } & \text { TCT TAGCA A G } & \text { G CACT T GA G A } \\ & \text { E45 } & \text { E46 } \\ \text { Mutant allele } & \text { TCT TAGCA A G } & \frac{\text { GACT C GCCTC }}{\text { E47 }}\end{array}$

B

$\begin{array}{ccc}\text { Exon } 46 & \text { c.6792 C>A } & \text { gDNA level } \\ \text { Normal allele } & \frac{G G A}{G} \frac{C C T}{P} \frac{G A C}{D} \frac{A C T}{T} \frac{T A C}{Y} \\ \text { Mutant allele } & \frac{G G A}{G} \frac{C C T}{P} \frac{G A C}{D} \frac{A C T}{T} \frac{T A A}{X}\end{array}$

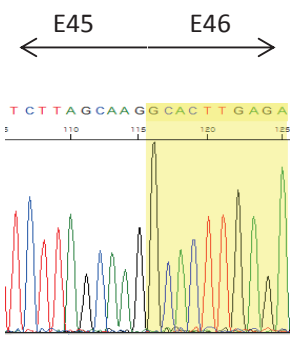

Control

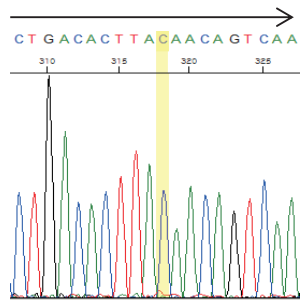

Control

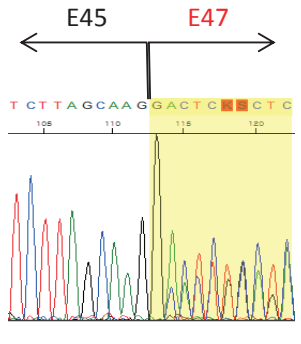

Patient

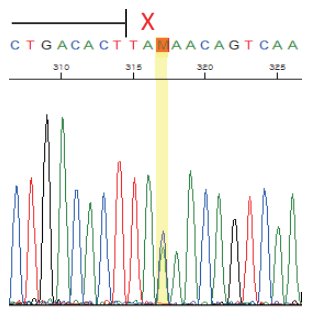

Patient

Fig. 2. Sanger sequencing results of exon 46 of NF1 in a patient. (A) Partial sequences of the complementary DNA (CDNA) of a control and a patient carrying a deletion of 102 nucleotides at the cDNA position c.6757_6858. (B) Genomic sequence of the exon-intron boundaries of exon 46 in a control individual and a patient. Nonsense mutation of cDNA at position c.6792C>A (p.Tyr2264*) detected in NF1. 
reported a proband Korean patient with NF1 who carries an allele with nonsense mutations at exon 46 (c. 6792C>A; p.Tyr2264*) in the gDNA, which led to the skipping of the whole of exon 46 (c.6757_6858del) in the cDNA. A mutation at position c. $6792 \mathrm{C}>\mathrm{A}$ or $\mathrm{C}>\mathrm{G}$ has been reported widely among NF1 patients worldwide, and this mutation is well known to cause exon skipping [4,6]. Moreover, a study has reported a mutation positioned at c.6792C $>$ A in two unrelated patients [7].

Here, we have described the first case in Korea of a proband with NF1 that carries an allele with an exon 46 deletion by ESE site mutation. Mutations at position c.6792C $>$ A were first described in two unrelated patients [5]. Although mutations at position c.6792C $>$ A are common, they have been reported to cause exon skipping in NF1 patients. Several research groups have shown that an ESE element spanning nucleotides c.6791_6795 (ACAAC motif) may be defective due to disruption of the ESE and may eliminate the ability of the positive transacting factor YB-1 to bind to this site.

The high risks of NF1 mutations have been attributed to the enormous size of the gene, the frequency of large deletions, and the common occurrence of random-splicing deficits that can be detected only by cDNA analysis. In addition, a large number of splicing aberrations are not affected by classical splicing, such as a "GT" splice donor or "AG" splice acceptor, and cannot create a new splice site. However, splicing aberrations may be influenced by alterations in the interaction between ESE and mRNA splicing factors [8]. Serine arginine-rich (SR) protein, an essential splicing factor, recognizes and moderates the ESE in pre-mRNA to normal splicing. Using an ESE prediction program, Zatkova et al. [4] showed disruption of ESE motifs in 6 mutations in wild-type and mutant $N F 1$, suggesting that disruption of ESE elements causes mutation-derived exon skipping in NF1 patients.

Mis-splicing caused by abrogation or aberrations in the normal function of ESEs has an important role in canonical exonic mRNA splicing. In fact, Liu et al. [8] described the mechanistic exon skipping caused by nonsense mutations in BRCA 1 in an in vitro study. In addition, Colapietro et al. [9] reported a patient with severe NF1 due to sequence alterations in ESE and subsequent skipping of exon 7.

Single-nucleotide polymorphisms in the exonic splicing coding region may affect the efficiency of non-canonical splicing, which could cause a severe penetrance of variants elsewhere in NF1. Although the existing data on the clinical manifestations of NF1 patients do not show any correlation or relationship to the specific genotypic variants, it is recommended that several patients should be screened and analyzed for the putative relationship with NF1 alterations in order to allow correlation of genotype and phenotype.

\section{Acknowledgements}

We thank our patient and her family members for consenting to and participating in this study. This work was supported by the National Research Foundation of Korea (NRF) grant funded by the Korea government (2007-0054214).

\section{References}

1. Danglot $G$, Régnier $V$, Fauvet $D$, Vassal G, Kujas M, Bernheim A. Neurofibromatosis 1 (NF1) mRNAs expressed in the central nervous system are differentially spliced in the 5 ' part of the gene. Hum Mol Genet 1995;4:915-20.

2. Williams VC, Lucas J, Babcock MA, Gutmann DH, Korf B, Maria BL. Neurofibromatosis type 1 revisited. Pediatrics 2009;123:124-33.

3. Friedman JM, Birch PH. Type 1 neurofibromatosis: a descriptive analysis of the disorder in 1,728 patients. Am J Med Genet 1997;70:138-43.

4. Zatkova A, Messiaen L, Vandenbroucke I, Wieser R, Fonatsch C, Krainer $A R$, et al. Disruption of exonic splicing enhancer elements is the principal cause of exon skipping associated with seven nonsense or missense alleles of NF1. Hum Mutat 2004;24:491-501.

5. Robinson PN, Böddrich A, Peters $H_{1}$ Tinschert S, Buske A, Kaufmann D, et al. Two recurrent nonsense mutations and a 4 bp deletion in a quasisymmetric element in exon 37 of the NF1 gene. Hum Genet 1995;96:958.

6. Hernández-Imaz E, Campos B, Rodríguez-Álvarez FJ, Abad O, Melean G, Gardenyes J, et al. Characterization of NF1 allele containing two nonsense mutations in exon 37 that segregates with neurofibromatosis type 1. Clin Genet 2013;83:462-6.

7. Ars E, Serra E, García J, Kruyer H, Gaona A, Lázaro C, et al. Mutations affecting mRNA splicing are the most common molecular defects in patients with neurofibromatosis type 1. Hum Mol Genet 2000;9:237-47.

8. Liu HX, Cartegni L, Zhang MQ, Krainer AR. A mechanism for exon skipping caused by nonsense or missense mutations in BRCA1 and other genes. Nat Genet 2001;27:55-8.

9. Colapietro P, Gervasini C, Natacci F, Rossi L, Riva P, Larizza L. NF1 exon 7 skipping and sequence alterations in exonic splice enhancers (ESEs) in a neurofibromatosis 1 patient. Hum Genet 2003;113:551-4. 